\title{
Disinfection Properties of Conventional White LED Illumination and Their Potential Increase by Violet LEDs for Applications in Medical and Domestic Environments
}

\author{
Jule Buehler ${ }^{1}$, Florian Sommerfeld ${ }^{1}$, Tobias Meurle', \\ Katharina Hoenes ${ }^{1}$, Martin Hessling ${ }^{1 *}$ \\ 1 Institute of Medial Engineering and Mechatronics, Ulm University of Applied Sciences, Germany \\ * Corresponding author's email: martin.hessling@thu.de
}

\begin{abstract}
The antimicrobial impact of visible violet and blue light has been known for more than a century but hardly been applied for purposeful pathogen reduction or prevention. The disinfecting properties of wide-spread warm-white and cool-white light emitting diodes (LEDs) are investigated by irradiation of staphylococci with different LEDs and varying doses. Additionally, the combination of a white and a violet LED illumination is examined. Both white LEDs exhibit an antimicrobial effect, which seems to be dominated by the blue parts of the LED emissions. Unfortunately, the antimicrobial effect is weak in realistic illumination applications. Additional violet LEDs can significantly enhance this impact without a large change in human color perception. This allows reasonable applications in certain medical and domestic environments without endangering humans.
\end{abstract}

Keywords: antimicrobial, visible light, illumination, white, violet, LEDs, staphylococci.

\section{INTRODUCTION}

The disinfection properties of short-wavelength visible light were discovered more than 100 years $[1,2]$. They have mostly been ignored because, compared to disinfection with ultraviolet (UV) radiation as emitted by mercury vapor lamps, high doses are required that have long been difficult to achieve in technically feasible times. Unfortunately, UV radiation also affects human cells and might lead to skin cancer, photokeratitis and cataratogenesis, so another kind of antimicrobial radiation, which is less harmful to humans, would be desirable. The development of powerful violet and blue light emitting diodes (LEDs) in recent years has restarted research into the antimicrobial properties of short-wavelength visible light. By now, it has been demonstrated that all investigated bacteria, fungi and probably even viruses can be inactivated if the irradiation dose is sufficiently $[3 \div 8]$.
The basics of the mechanism of action are understood meanwhile and schematically illustrated in Figure 1. Naturally occurring photosensitizers (PS) such as porphyrins and flavins absorb violet or blue light and generate so-called reactive oxygen species (ROS) in the presence of oxygen. These are radicals that attack virtually all relevant structures in the cell and can thus lead to its death $[3 \div 5,9 \div 12]$.

Maclean and coworkers successfully investigated the antimicrobial effect of violet light in hospital environments in several studies $[13 \div 15]$. Violet light with a wavelength of about $405 \mathrm{~nm}$ has a significantly stronger antimicrobial effect compared to blue light [6], and is at the same time better tolerated by human cells than ultraviolet radiation [16]. However, violet light generates effects similar to black light on fluorescent materials and can therefore be perceived as irritating and interferes with the viewer's color perception. Therefore, Gillespie et al. have proposed a white LED illumination 


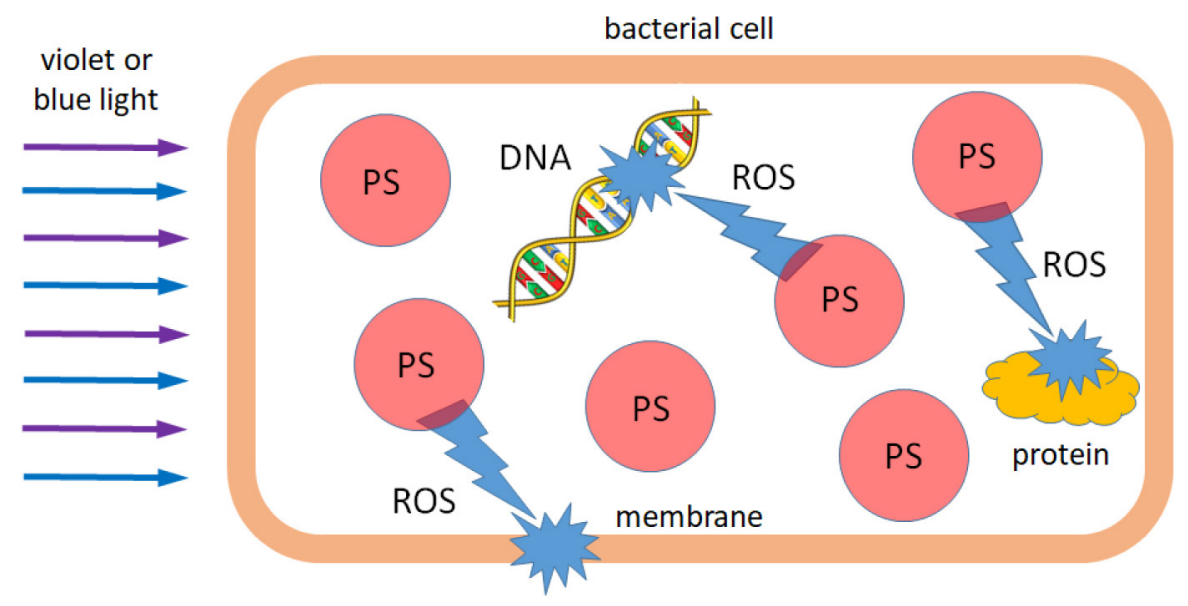

Fig. 1. Scheme of the disinfection mechanism of blue and violet light for bacteria (PS: Photosensitizer, ROS: reactive oxygen species, DNA: deoxyribonucleic acid)

composed of pulsed violet, green, yellow, and red LEDs [17]. This white illumination demonstrated improved color rendering and a simultaneous disinfecting effect on the clinical relevant pathogens Staphylococcus aureus and Pseudomonas aeruginosa.

Rohan et al. and Rutala et al. performed similar successful studies in hospital environments with $405 \mathrm{~nm}$ LEDs $[18,19]$. Rutala et al. [19] investigated the antimicrobial effect of white light with a noticeable staphylococci reduction even for low irradiation doses. These results fit to an own study, in which we investigated the disinfecting properties of a touch screen, and observed that a highly luminous blue screen was capable of pronounced bacterial reduction, while a white luminous screen, for which the light was a combination of red, green, and blue emissions, was even slightly more effective [20].

The study presented here aims to:

1. Investigate the disinfecting properties of commercial warm-white and cool-white LEDs, which both consist of blue LEDs and phosphorescent layers, in comparison to the antimicrobial properties of pure blue LEDs and whether green, yellow or red light portions of white LEDs deliver a significant contribution to the disinfection performance.

2. Furthermore, the question is addressed whether it is possible to combine a conventional white LED illumination with violet LEDs to increase the antimicrobial effect without changing the perceived color impression particularly. This would pose a significant technical improvement to the approach of Gillespie et al. [17]. The approach seems to be realistic, since the human eye sensitivity at $405 \mathrm{~nm}$, a typical wavelength of violet LEDs, is about a factor of 1000 lower than in the spectral range $520-590 \mathrm{~nm}$, in which conventional white LEDs emit to a large extent.

\section{MATERIALS AND METHODS}

\section{Irradiation Setup}

The following high-power LEDs were employed for the irradiation experiments: a warm-white LED type CXB1512-0000000F0HN430G of Cree (Durham, North Carolina (USA)), a cool-white LED type Cree CXB1310-0000-000F0HN265E, a blue LED type Cree XPEBRY-L1-0000-00Q01, and a violet LED type LZ1-00UB00 of LEDengin (San Jose, California (USA)).

The white and blue LEDs were selected to have similar peak wavelengths in the blue spectral region around $450 \mathrm{~nm}$. For the comparison of the antimicrobial impact of the warm-white, cool-white, and blue LED, the irradiance was adjusted using a DT-Blue $475 \mathrm{~nm}$ short-pass filter from Qioptiq (Goettingen, Germany) so that the blue component of the irradiance was $6.3 \mathrm{~mW} /$ $\mathrm{cm}^{2}$ for all white and blue LEDs. In this way, it could be investigated whether only the blue emissions are responsible for the potential antimicrobial effect of white LEDs.

For the experiments on the additional effect of violet LEDs, the irradiances of the 
warm-white LED and the violet LED were adjusted so that the irradiance in the violet-blue spectral region (below $480 \mathrm{~nm}$ ) was $5 \mathrm{~mW} /$ $\mathrm{cm}^{2}$. The spectra of the applied LEDs and the potentially involved bacterial photosensitizers can be found in Figure 2. The homogeneous irradiation of the bacterial samples was achieved with a pyramid-shaped mirror arrangement as described previously $[7,21]$. Because of the potential influence of the violet LED on human color perception color coordinates, correlated color temperature and color rendering indices (CRI) of the warm-white LED, the violet LED and the combination of both were calculated.

\section{Microbiological Experiments}

Since it is restricted to work with pathogens in the available laboratory, Staphylococcus carnosus (DSM 20501) was selected as a non-pathogenic bacterium that is related to the methicillinresistant Staphylococcus aureus (MRSA), which is notorious in hospitals but slightly less sensitive to visible light [7]. Concentrations in the range of $10^{6}$ bacteria per $\mathrm{ml}$ were prepared in phosphatebuffered saline solution (PBS) and $3 \mathrm{ml}$ were irradiated in glass beakers at a temperature of approximately $25^{\circ} \mathrm{C}$. At the beginning and during the course of irradiation, $100 \mu \mathrm{l}$ samples were taken and plated out on agar plates at different dilution levels. Surviving bacteria showed up after $24 \mathrm{~h}$ at $37^{\circ} \mathrm{C}$ in the incubator as visible and countable colonies, from which the bacterial concentration at the time of sampling could be calculated. As a control, non-irradiated bacterial samples were also always examined in parallel to the irradiation experiment. Due to the known high variability of such microbiological experiments, each individual sample was plated out at least three times at each dilution level and each series of experiments was performed three times separately.

\section{RESULTS}

The results of the photoinactivation of $S$. carnosus by warm-white, cold-white and blue LED emission are presented in Figure 3 as a function of the blue irradiation dose. At first glance, warm-white seems to have a slightly stronger antimicrobial impact than the blue and cold-white illumination, but the error bars reveal a high variation in the underlying results of the single experiments and one should keep in mind, that the given irradiance is just the blue part of the LED emission. The results of both white LEDs are close to the results of the blue LED. This indicates that the disinfecting effect of the white LEDs is predominantly caused by their blue emission components.

The blue irradiation dose necessary for 1 and 3 log-reductions ( $90 \%$ and $99.9 \%$ reduction) of S. carnosus is about $130 \mathrm{~J} / \mathrm{cm}^{2}$ and $210 \mathrm{~J} / \mathrm{cm}^{2}$, respectively. The contribution of the blue part of the cool-white LED emission is approximately $24 \%$ of its total irradiance. For the warm-white

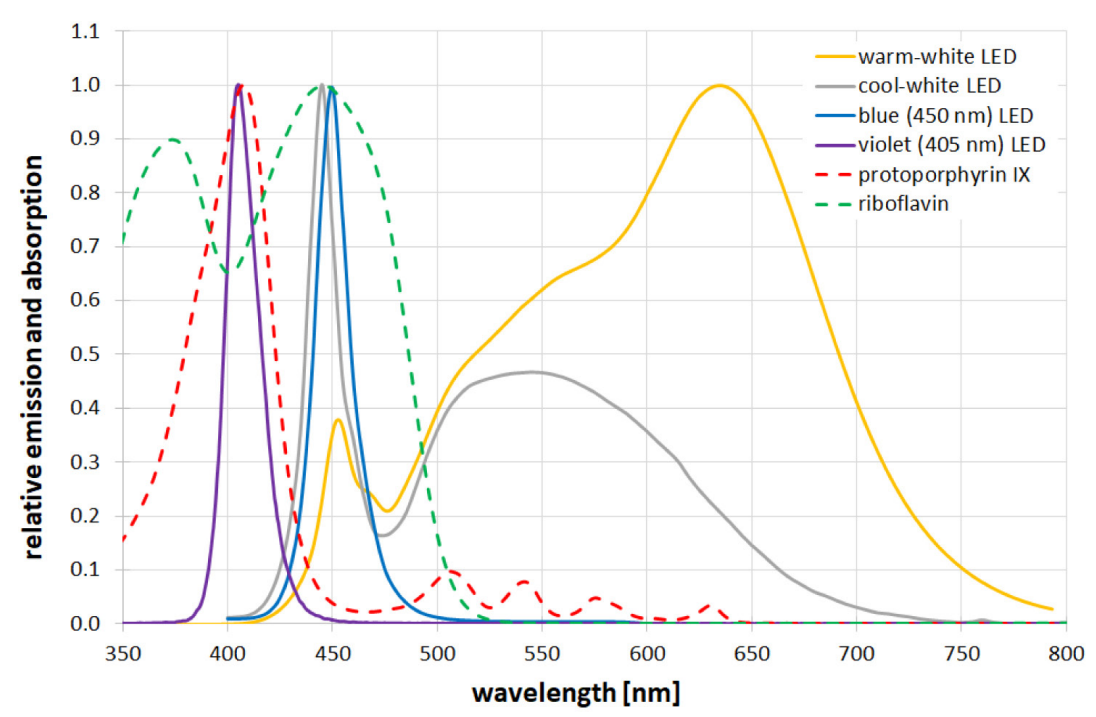

Fig. 2. Relative emission spectra of the selected warm-white, cool-white, blue and violet LEDs and the relative absorption spectra of protoporphyrin IX and riboflavin 


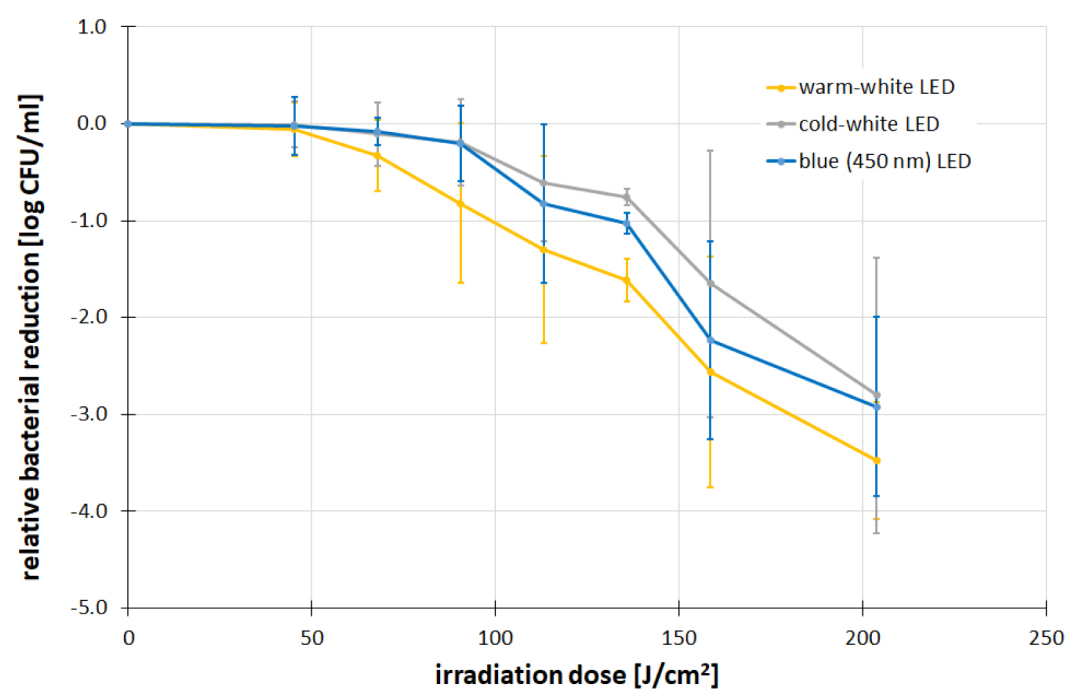

Fig. 3. S. carnosus concentration as a logarithmic relative representation of colony forming units (CFU) per $\mathrm{ml}$ as a function of the blue irradiation dose. Each value represents the average of at least three independent experiments and the error bars depict the standard deviation of these single measurements

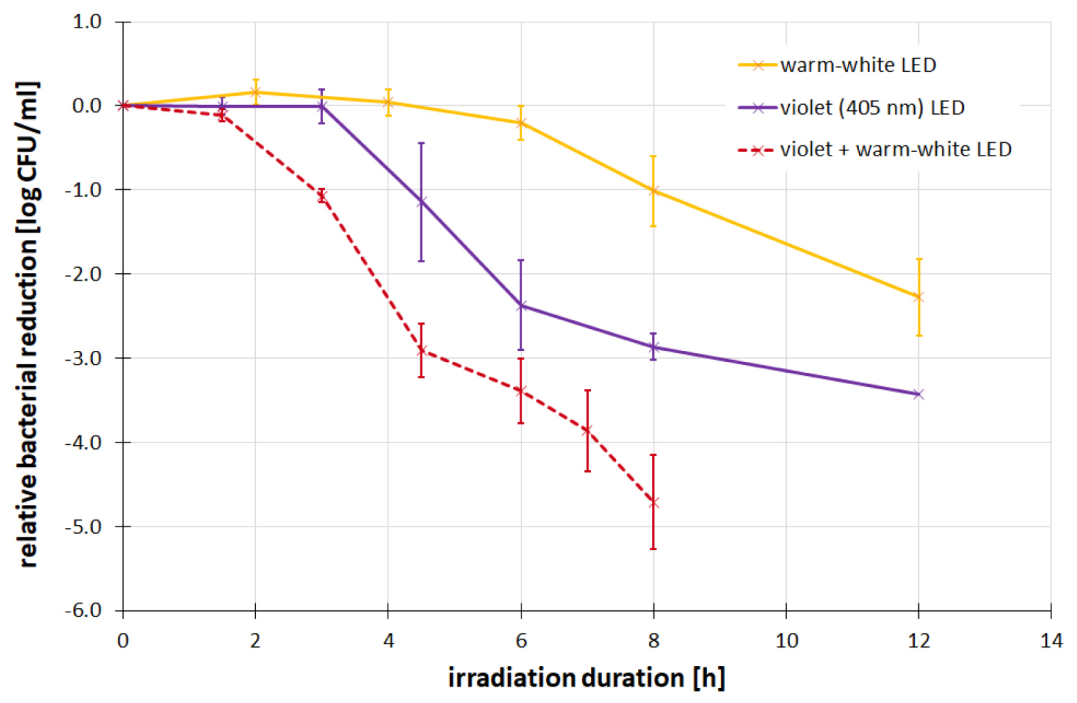

Fig. 4. S. carnosus concentration as a logarithmic relative representation of colony forming units (CFU) per $\mathrm{ml}$ as a function of irradiation duration. Each value represents the average of at least three independent experiments and the error bars depict the standard deviation of these single measurements

LED this blue fraction is three times lower with a percentage of about $8 \%$. Therefore, a cool-white LED should have an approximately three times higher disinfecting impact at the same total irradiance than a warm-white one.

The antimicrobial effect of the warm-white and the violet LED, and the combination of both is given in Figure 4. A 1 log-reduction for staphylococci is reached after about $8 \mathrm{~h}, 4.3 \mathrm{~h}$ and 2.9 $\mathrm{h}$ for the warm-white LED, the violet LED and the LED combination. Concerning the violet irradiation doses the 1 and 3 log-reductions required approximately 77 and $162 \mathrm{~J} / \mathrm{cm}^{2}$.

Table 1 and Figure 5 illustrate the human color impression of the warm-white and the violet LED, and the combination of both. There is almost no difference in the color coordinates, correlated temperature and color rendering between the white LED and the combination with the violet LED. Even a hypothetic tenfold increase in the violet irradiance would only lead to moderate changes in the total color perception. 
Table 1. CIE (Commission Internationale de l'Éclairage) xy color coordinates, correlated color temperatures (CCT) and color rendering indices (CRI) calculated for spectra of the warm-white, the violet, the combined warm-white and violet LED and an additional hypothetic combination of the warm-white LED with a ten times stronger violet one

\begin{tabular}{|c|c|c|c|c|}
\hline LED & CIE x & CIE y & CCT [K] & CRI \\
\hline Violet & 0.172 & 0.028 & - & - \\
\hline Warm-white & 0.451 & 0.415 & 2880 & 98 \\
\hline Warm-white \& violet & 0.445 & 0.406 & 2901 & 98 \\
\hline Warm-white \& 10x violet & 0.397 & 0.341 & 3256 & 92 \\
\hline
\end{tabular}

\section{DISCUSSION}

The results reveal that warm-white and coolwhite LEDs exhibit antimicrobial properties, which are predominantly caused by their blue components and cool-white LEDs are supposed to be more efficient for the same total irradiance.

Operating theatres in hospitals represent an obvious field of application. Pathogen reduction is of great importance there and, at the same time, high illuminance levels of $10000-100000$ lu$\mathrm{men} / \mathrm{m}^{2}$ are required [23], which would be a maximum of up to $10 \mathrm{~lm} / \mathrm{cm}^{2}$. If this illuminance is generated just with the warm-white LED, this corresponds to a blue irradiance of approx. 2.3 $\mathrm{mW} / \mathrm{cm}^{2}$. A $90 \%$ staphylococci reduction thus requires about $15.7 \mathrm{~h}$, which seems to be of limited

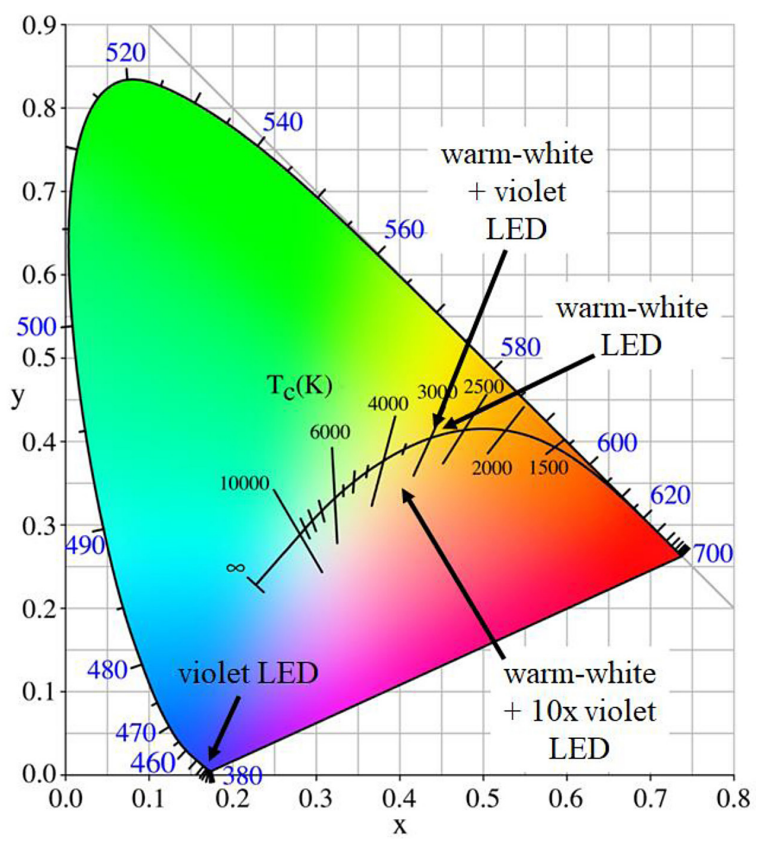

Fig. 5. Representation of the different tested LED illuminations in a CIE color diagram. Included is a hypothetic additional combination of the warm-white LED and a 10x stronger violet LED (Modified according to [22]) use in this form even for an automatic overnight disinfection. In combination with the violet LED (additional $2.3 \mathrm{~mW} / \mathrm{cm}^{2}$ of $405 \mathrm{~nm}$ ), however, this time would already decrease to about $8.4 \mathrm{~h}$. The simulated combination of a warm-white LED $\left(2.3 \mathrm{~mW} / \mathrm{cm}^{2}\right.$ of $\left.450 \mathrm{~nm}\right)$ and a $10 \mathrm{x}$ stronger violet LED $23 \mathrm{~mW} / \mathrm{cm}^{2}$ of $405 \mathrm{~nm}$ ), a further reduction in the range of about $1 \mathrm{~h}$ is feasible and this does not have to be the limit, if the violet irradiation is further increased.

In these considerations, 1 log-reduction data of S. carnosus in liquids were presupposed: $77 \mathrm{~J} / \mathrm{cm}^{2}$ for violet light and $130 \mathrm{~J} / \mathrm{cm}^{2}$ for blue light. These assumptions are rather pessimistic. In our own previous investigations, the medically more significant pathogen S. aureus turned out to be more sensitive to light for both spectral ranges [7]. Applying $405 \mathrm{~nm}$ in a hospital setting, Rutala et al. observed a $90 \%$ staphylococcus reduction on surfaces for a dose as little as $14.5 \mathrm{~J} / \mathrm{cm}^{2}$ [19], and Gillespie et al. reported that less than $4 \mathrm{~J} / \mathrm{cm}^{2}$ were needed for a log-reduction in their $S$. aureus experiments [17].

In addition to hospital and healthcare settings, applications of white-violet combinations in domestic environments such as bathrooms or kitchens are also conceivable. For example, campylobacter, food pathogens that frequently cause intestinal infections, are very sensitive to violet light and require only a dose of $3.5 \mathrm{~J} / \mathrm{cm}^{2}$ of 405 $\mathrm{nm}$ irradiation for a $90 \%$ reduction [24]. Technically, a kitchen work area could be equipped with an appropriate antimicrobial lighting. Just $10 \mathrm{~W}$ of violet illumination could generate an irradiance of about $4 \mathrm{~mW} / \mathrm{cm}^{2}$ on an area of $60 \times 40$ $\mathrm{cm}^{2}$, and thus reduces the previously mentioned campylobacters by $90 \%$ within 15 min. Applications in which white and violet do not necessarily radiate simultaneously all the time are also conceivable. For example, it would be imaginable to have a white-violet shower illumination that only shines white during the shower process and then automatically switches to violet-white or violet for a selected duration, afterwards. 


\section{CONCLUSION}

White LEDs and the combination of white and violet LEDs do not replace UV irradiation in its antimicrobial effect and short application duration, but allow many possible approaches for pathogen reduction and infection prevention in medical and domestic environments in the immediate vicinity of humans.

\section{REFERENCES}

1. Downes A, Blunt TP. Researches on the Effect of Light upon Bacteria and other Organisms. Proceedings of the Royal Society of London. 1877;26:488500. doi:10.1098/rspl.1877.0068.

2. Ward HM. The Action of Light on Bacteria. III. Philosophical Transactions of the Royal Society B: Biological Sciences. 1894;185:961-86. doi:10.1098/rstb.1894.0020.

3. Ashkenazi H, Malik Z, Harth Y, Nitzan Y. Eradication of Propionibacterium acnes by its endogenic porphyrins after illumination with high intensity blue light. FEMS Immunology \& Medical Microbiology. 2003;35:17-24. doi:10.1111/j.1574695X.2003.tb00644.x.

4. Guffey JS, Wilborn J. In vitro bactericidal effects of 405-nm and 470-nm blue light. Photomed Laser Surg. 2006;24:684-8. doi:10.1089/ pho.2006.24.684.

5. Maclean M, MacGregor SJ, Anderson JG, Woolsey G. High-intensity narrow-spectrum light inactivation and wavelength sensitivity of Staphylococcus aureus. FEMS Microbiol Lett. 2008;285:227-32. doi:10.1111/j.1574-6968.2008.01233.x.

6. Hessling M, Spellerberg B, Hoenes K. Photoinactivation of bacteria by endogenous photosensitizers and exposure to visible light of different wavelengths - A review on existing data. FEMS Microbiol Lett. 2016;364:fnw270. doi:10.1093/ femsle/fnw270.

7. Hoenes K, Bauer R, Meurle T, Spellerberg B, Hessling M. Inactivation Effect of Violet and Blue Light on ESKAPE Pathogens and Closely Related Non-pathogenic Bacterial Species - A Promising Tool Against Antibiotic-Sensitive and Antibiotic-Resistant Microorganisms. Front Microbiol. 2020;11:612367. doi:10.3389/fmicb.2020.612367.

8. Vatter P, Hoenes K, Hessling M. Photoinactivation of the Coronavirus Surrogate phi6 by Visible Light. Photochem Photobiol. 2021;97:122-5. doi:10.1111/php.13352.

9. Feuerstein O, Ginsburg I, Dayan E, Veler D, Weiss EI. Mechanism of visible light phototoxicity on Porphyromonas gingivalis and Fusobacterium nu- cleatum. Photochem Photobiol. 2005;81:1186-9. doi:10.1562/2005-04-06-RA-477.

10. Amin RM, Bhayana B, Hamblin MR, Dai T. Antimicrobial blue light inactivation of Pseudomonas aeruginosa by photo-excitation of endogenous porphyrins: In vitro and in vivo studies. Lasers Surg Med. 2016;48:562-8. doi:10.1002/lsm.22474.

11. Plavskii VY, Mikulich AV, Tretyakova AI, Leusenka IA, Plavskaya LG, Kazyuchits OA, et al. Porphyrins and flavins as endogenous acceptors of optical radiation of blue spectral region determining photoinactivation of microbial cells. J Photochem Photobiol B. 2018;183:172-83. doi:10.1016/j. jphotobiol.2018.04.021.

12. Cieplik F, Spath A, Leibl C, Gollmer A, Regensburger J, Tabenski L, et al. Blue light kills Aggregatibacter actinomycetemcomitans due to its endogenous photosensitizers. Clin Oral Investig. 2014;18:1763-9. doi:10.1007/s00784-013-1151-8.

13. Maclean M, Macgregor SJ, Anderson JG, Woolsey GA, Coia JE, Hamilton K, et al. Environmental decontamination of a hospital isolation room using high-intensity narrow-spectrum light. J Hosp Infect. 2010;76:247-51. doi:10.1016/j.jhin.2010.07.010.

14. Bache SE, Maclean M, MacGregor SJ, Anderson JG, Gettinby G, Coia JE, Taggart I. Clinical studies of the High-Intensity Narrow-Spectrum light Environmental Decontamination System (HINSlight EDS), for continuous disinfection in the burn unit inpatient and outpatient settings. Burns: Journal of the International Society for Burn Injuries. 2012;38:69-76. doi:10.1016/j.burns.2011.03.008.

15. Maclean M, Booth MG, Anderson JG, Macgregor SJ, Woolsey GA, Coia JE, et al. Continuous decontamination of an intensive care isolation room during patient occupancy using $405 \mathrm{~nm}$ light technology. Journal of Infection Prevention. 2013;14:176-81. doi:10.1177/1757177413483646.

16. Yin R, Dai T, Avci P, Jorge AES, de Melo, Wanessa C M A, Vecchio D, et al. Light based anti-infectives: ultraviolet $\mathrm{C}$ irradiation, photodynamic therapy, blue light, and beyond. Curr Opin Pharmacol. 2013;13:731-62. doi:10.1016/j.coph.2013.08.009.

17. Gillespie JB, Maclean M, Wilson MP, Given MJ, MacGregor SJ. Development of an antimicrobial blended white LED system containing pulsed 405nm LEDs for decontamination applications. In: Raghavachari R, Liang R, Pfefer TJ, editors. SPIE BiOS; Saturday 28 January 2017; San Francisco, California, United States: SPIE; 2017. 100560Y. doi:10.1117/12.2250539.

18. Rohan A, Khan I, Yin D, Yang J. Passive Ceiling Light Disinfection System to Reduce Bioburden in an Intensive Care Unit. J Pediatr Intensive Care. 2019;8:138-43. doi:10.1055/s-0038-1676655.

19. Rutala WA, Kanamori H, Gergen MF, SickbertBennett EE, Sexton DJ, Anderson DJ, et al. Antimicrobial activity of a continuous visible light dis- 
infection system. Infect Control Hosp Epidemiol. 2018;39:1250-3. doi:10.1017/ice.2018.200.

20. Hessling M, Spellerberg B, Hönes K. Potential selfdisinfection capacity of touch screen displays. J Biophotonics. 2019;12:e201900118. doi:10.1002/ jbio.201900118.

21. Hoenes K, Stangl F, Sift M, Hessling M. Visible optical radiation generates bactericidal effect applicable for inactivation of health care associated germs demonstrated by inactivation of E. coli and B. subtilis using 405-nm and 460-nm light emitting diodes. In: Amelink A, Vitkin IA, editors; Sunday 21 June 2015; Munich, Germany: SPIE; 2015. p. 95400. doi:10.1117/12.2183903.
22. User Par. Planckian Locus. 2012. https://commons.wikimedia.org/wiki/File:PlanckianLocus. png. Accessed 2021.

23. DIN Deutsches Institut für Normung e.V. DIN EN 12464-1:2019-06, Licht und Beleuchtung Beleuchtung von Arbeitsstätten - Teil_1: Arbeitsstätten in Innenräumen; Deutsche und Englische Fassung prEN_12464-1:2019 2019. Berlin: Beuth Verlag GmbH. doi:10.31030/3045264.

24. Murdoch LE, Maclean M, MacGregor SJ, Anderson JG. Inactivation of Campylobacter jejuni by exposure to high-intensity $405-\mathrm{nm}$ visible light. Foodborne Pathog Dis. 2010;7:1211-6. doi:10.1089/ fpd.2010.0561. 